\title{
Estudio descriptivo-transversal del síndrome de Down en pacientes del departamento de Boyacá (Colombia)
}

\author{
Descriptive-Transversal Study of the Down \\ Syndrome in Patients from Boyacá (Colombia)
}

Fecha de Recepción: 26 - nov - 2013

Fecha de Aceptación: 18 - nov - 2014

\author{
E. J. Vergara Estupiñán a* \\ M. Forero-Castro * \\ J. I. Moreno Granados b
}

\begin{abstract}
Resumen
El síndrome de Down (SD) es la aneuploidía autosómica más común y la principal causa de retraso mental de origen genético; se presenta en uno de cada 650 a 1000 nacidos vivos y genera una importante disminución en la calidad y expectativa de vida de los afectados. El objetivo del estudio fue realizar un análisis descriptivo del comportamiento poblacional de algunas de las variables relacionadas con la ocurrencia del SD en el departamento de Boyacá, las cuales han sido convencionalmente estudiadas en otras poblaciones del país y del mundo. En 43 localidades del departamento de Boyacá se encuestaron 140 núcleos familiares con por lo menos un caso de SD. Se estudiaron variables cualitativas y cuantitativas, tales como edad materna y paterna, antecedentes familiares y estudios genéticos realizados en los pacientes. Se observó que, en el departamento, el examen de cariotipo no es rutinariamente utilizado como apoyo diagnóstico para el estudio de anomalías cromosómicas como el SD, debido al alto costo y al acceso limitado a esta prueba. El mayor número de casos con SD se presentó en madres con edades entre 30 y 35 años ( $27,8 \%$ del total de casos). En las familias con casos recurrentes de SD se descartó, mediante cariotipo, la heredabilidad de alguna anormalidad cromosómica; sin embargo, se desconoció la incidencia de un factor genético en los abortos espontáneos ocurridos en las familias afectadas. Es recomendable implementar asesorías genéticas que informen sobre el origen y riesgo de recurrencia de casos de SD en la familia. De igual manera, es importante fomentar estudios citogenéticos que apoyen el diagnóstico de pacientes que puedan estar afectados por anormalidades cromosómicas, teniendo en cuenta que desde el año 2011 se incluyeron las pruebas citogenéticas en el listado de pruebas diagnósticas disponibles para la población colombiana en el plan obligatorio de salud.
\end{abstract}

Palabras claves: Citogenética, Edad materna, Síndrome de Down.

\begin{abstract}
Down Syndrome (DS) is the most common autosomal aneuploidy and the main cause of mental retardation from genetic origin, it occurs in 1 in every 650 1,000 live births, and the syndrome generates a significant
\end{abstract}

a Facultad de Ciencias, Escuela de Ciencias Biológicas, Grupo de Estudios en Genética y Biología Molecular(GEBIMOL), Universidad Pedagógica y Tecnológica de Colombia, Tunja, Colombia.

* Autor de correspondencia: ejaviervergara@gmail.com

b Escuela de Medicina, Facultad de Ciencias de la salud, Universidad Pedagógica y Tecnológica de Colombia, Tunja, Colombia. 
decline in the affected's life expectancy and quality. The aim of the study was to perform a descriptive analysis about the population behavior in front of some variables related to the DS occurrence in the Boyacá department, which have conventionally been studied in other locations of the country and the world. One hundred forty family units were surveyed with at least one case of DS in forty-three locations in the Boyacá department. Qualitative and quantitative variables such as maternal and paternal age, family history and genetic studies in patients were studied. It was noted that in the department, a karyotype test is not routinely used as diagnostic support for the study of chromosomal abnormalities such as DS, due to their high cost and limited access. The largest number of DS cases occurred in mothers aged 30-35 years ( $27.8 \%$ of all cases). In families with recurrent DS cases was ruled out by karyotype test that an inherited chromosomal abnormality could be responsible for this condition, however, it was unknown whether spontaneous abortion were influenced by genetic factor in the affected families. Genetic counseling is recommended to inform about the origin and risk of recurrent DS cases in the family. Similarly, it is important to encourage cytogenetic studies supporting the diagnosis of patients who may be affected by chromosomal abnormalities, taking into account that since 2011 the cytogenetic tests were included in the list of diagnostic tests available for the Colombian population in the Mandatory Health Plans.

Keywords: cytogenetics, maternal age, Down syndrome

\section{INTRODUCCIÓN}

El síndrome de Down (SD), o trisomía 21, es la cromosomopatía más frecuente en humanos, y es la primera responsable del retardo mental en el mundo en lo que refiere a causas genéticas [1]; afecta aproximadamente a 1/650-1000 nacidos vivos, y presenta una heterogeneidad en el comportamiento poblacional de sus variables relacionadas [2]. Estudios poblacionales, como los registrados por Carothers et al. [3], muestran que la tasa de variación del SD puede cambiar en un $25 \%$, según la población y la estructura de la edad materna [4].

En Colombia se han hecho estudios sobre las principales variables relacionadas con los casos de SD en ciudades como Manizales [5], Cali [6], Bogotá [7] y Pasto [8]; Boyacá carece de estudios poblacionales y epidemiológicos del SD a cualquier nivel. Según lo informado por la secretaría de salud del departamento de Boyacá y las fundaciones más destacadas del departamento que acogen este tipo de población, no se encuentra disponible un registro organizado y actual, que describa las principales variables relacionadas con la aparición de casos de SD [9].

Se ha observado que la estructura de la edad materna y paterna influye decisivamente en la aparición de casos de SD [8]. Conocer el comportamiento de la edad en los padres gestantes permite a las autoridades implementar acciones con el ánimo de disminuir el impacto de la patología en la esfera psicológica y socioeconómica de la región [10].
El apoyo diagnóstico de este síndrome a nivel prenatal y posnatal se brinda mediante el examen de cariotipo [11], que permite identificar el tipo de anormalidad cromosómica; este estudio aporta herramientas para los genetistas a la hora de establecer el posible riesgo de aparición de nuevos casos de SD y descartar el factor genético en los abortos espontáneos ocurridos en la familia $[12,13]$. No obstante, no hay documentación que permita saber si las familias afectadas por casos $\mathrm{SD}$ en el departamento de Boyacá han accedido a este tipo de servicios [12]. Este trabajo tuvo como objetivo realizar un análisis descriptivo-transversal de algunas de las variables asociadas con la ocurrencia del SD en 43 localidades del departamento de Boyacá, constituyéndose en un primer acercamiento al estudio del comportamiento poblacional del SD en Boyacá.

\section{MATERIALES Y MÉTODOS}

\subsection{Población y muestra.}

Se realizó un estudio descriptivo transversal que incluyó una población de 140 pacientes colombianos con diagnóstico confirmado de SD, residentes en diferentes localidades del departamento de Boyacá. Para obtener la muestra de estudio se consultó la base de datos de estudiantes afectados con $\mathrm{SD}$ en las instituciones educativas públicas del departamento de Boyacá, suministradas por la Secretaria de Educación del Departamento; además se consultaron fundaciones públicas y privadas de pacientes con discapacidad cognitiva 
dentro de las cuales se encontraron: Fundown, Superar, Asdepal, UAI Programa RBC, Aula Tecnológica Culturama, UAI Programa RBC y Fundiferente. De esta manera se obtuvo un listado de personas boyacenses con SD, los cuales fueron contactados e informados del alcance de la investigación.

El estudio fue previamente aprobado por el Comité de Ética de la Universidad Pedagógica y Tecnológica de Colombia con sede en Tunja, mediante acta de aprobación $\mathrm{N}^{\circ} 1$ del 21 de Junio de 2010, y se rigió bajo la Declaración de Helsinki de la Asociación Médica Mundial y la Resolución 8430 de 1993 del Ministerio de Salud de Colombia. Los criterios de inclusión para participar del estudio fueron: pacientes con SD clínicamente confirmado, de cualquier sexo y edad, residentes del departamento de Boyacá, cuyos acudientes o representantes legales accedieran a participar del estudio mediante la firma del consentimiento informado.

\subsection{Indagación de antecedentes.}

Durante el periodo de agosto de 2009 a mayo del 2011 se aplicaron encuestas a 140 acudientes o representantes legales de familias que contaran con uno o más casos de SD clínicamente confirmados; las encuestas fueron estructuradas con base a las siguientes variables de conocida influencia sobre el SD: demográficas (sexo, edad, lugar y fecha de nacimiento) [13][14] [15], clínicas: (edad materna y paterna al momento de la gestación, otros casos de SD en el núcleo familiar por línea materna y paterna, grado de parentesco entre estos casos) [16][17] [18], diagnósticas (examen de cariotipo en la persona, examen de cariotipo en los padres) [19] y obstétricas (número de partos, número de abortos espontáneos, causas clínicas de los abortos espontáneos) [20] [21]. Se hicieron los árboles genealógicos familiares de mínimo tres generaciones en el programa CYRILLIC 2.1 ${ }^{\circledR}$, para el análisis directo de las genealogías.

\subsection{Estudio citogenético por bandeo GTG}

Con el fin de descartar que una anormalidad cromosómica heredada por línea germinal fuera la responsable de esta condición, se les realizó el examen de cariotipo con bandeo GTG a las familias que presentaron más de un caso de SD. El estudio citogenético se hizo acorde con el protocolo de Ikeuchi [22], y los resultados se reportaron siguiendo los criterios del ISCN 2013 (International System for Human Cytogenetic Nomenclature) [23].

\subsection{Asesoramiento genético y difusión de los resultados}

A las familias encuestadas se les dió una charla informativa sobre el SD, con el propósito de describir lo aspectos básicos relacionados con la aparición y seguimiento de la patología, los resultados citogenéticos se reportaron mediante interconsulta de genética médica; además, se asesoró a las personas y sus familias sobre los resultados referentes a la patogénesis del $\mathrm{SD}$, su heredabilidad, los riesgos de recurrencia, riesgos de padecer el SD en la familia y la toma de decisiones informadas. Los principales resultados de los estudios fueron socializados ante las fundaciones e instituciones que cooperaron con el proyecto en aras de fortalecer el vínculo institucional entre la academia y sociedad civil, en el abordaje de esta patología.

\subsection{Análisis de datos.}

Se analizaron las frecuencias y los porcentajes de variables como localidad de residencia, género y edad del paciente; se aplicó la prueba de bondad de ajuste de Kolmogorov Smirnov donde se evaluó las distribuciones teóricas de probabilidad Normal, Uniforme, Poisson y Exponencial, para las variables: edad de los pacientes, de las madres y de los padres en el momento de la gestación; para dicho análisis se utilizó un intervalo de confianza del 95\%. Se empleó el Análisis Discriminante en busca de funciones discriminantes a partir de las variables independientes para clasificar a los individuos según los valores de las éstas; las variables que hicieron parte del análisis fueron: antecedentes de SD y de abortos espontáneos en la familia. Este análisis permitió agrupar y caracterizar la población según características comunes. Los análisis fueron aplicados mediante los programas estadísticos SPSS Statistics 17,0, ${ }^{\circledR}$ y Statistics 17.0. y Statgraphics Plus 5.0.3. 


\section{RESULTADOS}

Las familias y los pacientes que hicieron parte del estudio se distribuyeron en 43 localidades del departamento de Boyacá, como se reporta en la tabla 1. En lo que respecta a los pacientes, el $49.3 \%(69 / 140)$ fueron de sexo femenino y el $50.7 \%(70 / 140)$ de sexo masculino. La mediana de edad de hombres fue de 13 años (rango 3-38 años) y de mujeres de 11 años (rango 1-33 años).

Tabla 1. Lista total de municipios donde fueron contactados los pacientes, indicando número y porcentaje de afectados con SD.

\begin{tabular}{|c|c|c|}
\hline Municipio & Número de pacientes & Porcentaje de pacientes \\
\hline Tunja & 18 & 12.9 \\
\hline Sogamoso & 17 & 12.1 \\
\hline Duitama & 13 & 9.3 \\
\hline Moniquirá & 7 & 5.0 \\
\hline Garagoa & 6 & 4.3 \\
\hline Aquitania & 5 & 3.6 \\
\hline Boavita & 5 & 3.6 \\
\hline Chiquinquirá & 5 & 3.6 \\
\hline Guayatá & 5 & 3.6 \\
\hline La Capilla & 5 & 3.6 \\
\hline Sativa Norte & 4 & 2.9 \\
\hline Belén & 3 & 2.1 \\
\hline Soatá & 3 & 2.1 \\
\hline Socha & 3 & 2.1 \\
\hline Susacón & 3 & 2.1 \\
\hline Tota & 3 & 2.1 \\
\hline Otros* & $35^{*}$ & 25 \\
\hline Total & 140 & 100.0 \\
\hline
\end{tabular}

* Corresponden a municipios con 1 y 2 pacientes afectados.

La mediana de edad materna al momento de la gestación fue de 30 años, entre un rango de edades de 16 y 46 años, mientras que la mediana de edad paterna fue de 33 , entre un rango de edades de 20 a 62 . En la figura 1 se ilustra la relación entre la edad materna y la paterna al momento de la gestación, con respecto al número de casos de SD registrados para un quinquenio (intervalo de edad de cinco años).

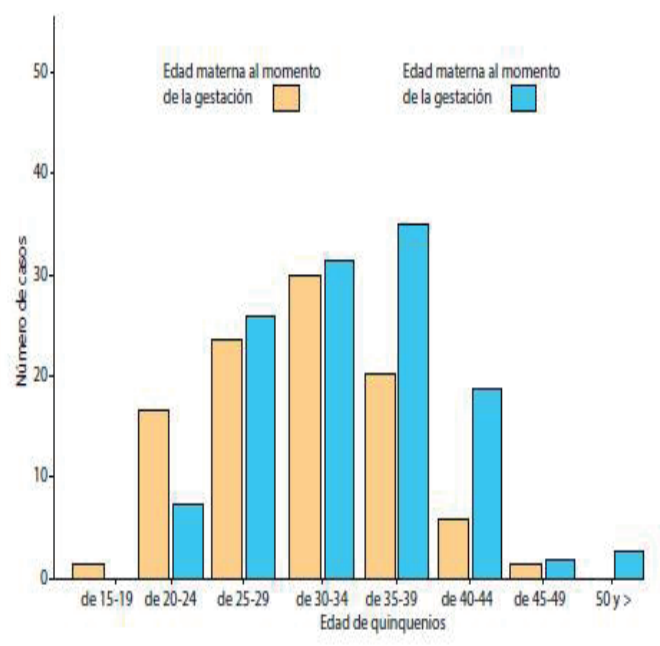

Figura 1. Comportamiento de la edad paterna y materna al momento de la gestación, registrada en intervalos de edad de cinco años, respecto a la aparición de casos de SD. Se puede reconocer que la mayor incidencia de SD para la edad materna se registró en el intervalo de 30 a 34 años, y para la edad paterna se registró en el de 35-39 años. 
En un total de 15 familias (10,71\%) se evidenciaron casos de abortos espontáneos en el núcleo familiar o en algún familiar en primer grado de parentesco, los porcentajes de las causas y tipos de abortos se muestran en la tabla 2 .

Tabla 2. Resultados de los antecedentes de abortos espontáneos en las familias encuestadas. Se registraron características que agrupan la familia, la edad materna en el momento de la pérdida, el porcentaje de las causas y el tipo de aborto.

\begin{tabular}{|c|c|c|c|c|c|c|c|c|c|c|}
\hline \multirow[b]{2}{*}{$\begin{array}{c}\text { Características de la } \\
\text { familia en la que se } \\
\text { produjo el aborto }\end{array}$} & \multirow[b]{2}{*}{$\begin{array}{l}\text { Número } \\
\text { de casos y } \\
\text { porcentaje }\end{array}$} & \multicolumn{2}{|c|}{ Edad materna } & \multicolumn{4}{|c|}{ (\%) Causas del aborto } & \multicolumn{3}{|c|}{ (\%) Tipo de aborto } \\
\hline & & 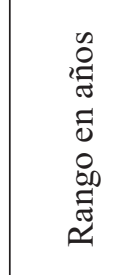 & 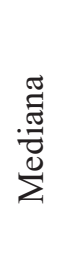 & 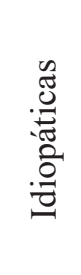 & 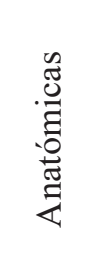 & $\begin{array}{l}0 \\
0 \\
.00 \\
.00 \\
00 \\
.00 \\
0 \\
0 \\
0 \\
0\end{array}$ & 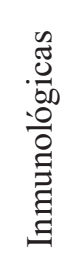 & 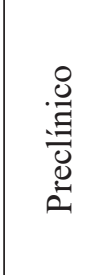 & 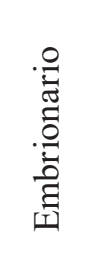 & 总 \\
\hline $\begin{array}{l}\text { Antecedente de solo un } \\
\text { (1) } \mathrm{AE}^{*}\end{array}$ & $5 \%(7 / 140)$ & $26-38$ & 33 & 71,4 & 14,3 & 0 & 14,3 & 57,1 & 28,6 & 14,3 \\
\hline $\begin{array}{l}\text { Antecedentes de } \mathrm{AE}^{*} \\
\text { en familiares de primer } \\
\text { grado de parentesco por } \\
\text { línea paterna o materna }\end{array}$ & $3,57 \%(8 / 140)$ & $26-36$ & 32 & 60 & 40 & 0 & 0 & 40 & 40 & 20 \\
\hline $\begin{array}{l}\text { Antecedentes de } \mathrm{AE}^{*}, \\
\text { en familiares de primer } \\
\text { grado de parentesco por } \\
\text { línea materna }\end{array}$ & $2,14 \%(3 / 140)$ & $33-39$ & 35 & 66.6 & 0 & 33,3 & 0 & 66,6 & 33,3 & 0 \\
\hline Promedio ponderado & $\begin{array}{l}10,71 \% \\
(15 / 140)\end{array}$ & 26-39 & 33 & 66 & 18,1 & 11,1 & 4,7 & 54,5 & 33,9 & 11,4 \\
\hline
\end{tabular}

*AE Aborto espontáneo

En la prueba de bondad de ajuste Kolmogorov Smirnov se obtuvo que únicamente las variables edad materna al momento de la gestación y edad paterna al momento de la gestación siguieron una distribución normal de probabilidad obteniendo un valor $Z$ de 0,733 y un nivel crítico de 0,655 y 0,172 que fueron mayores a 0.05 .
Se agrupó y caracterizó la muestra de estudio mediante la presencia de las variables abortos espontáneos y repeticiones de SD en un núcleo familiar, mediante un análisis de las genealogías graficadas con el programa CYRILLIC 2.1®, y complementado con el análisis discriminante aplicado mediante programa SPSS Statistics 17,0, ( ) (Tabla 3).

Tabla 3. Resumen y agrupación de las 140 familias encuestadas según las variables asociadas con el SD, frente a los abortos espontáneos (AE) y repetición de casos de SD en la familia.

\begin{tabular}{|l|c|c|}
\hline \multicolumn{1}{|c|}{ Tipo de familias } & Número de casos & $\begin{array}{c}\text { Porcentaje } \\
\text { de casos }\end{array}$ \\
\hline Familias con un solo caso de SD*, sin AE† & $(119 / 140)$ & $85 \%$ \\
\hline $\begin{array}{l}\text { Parejas con un caso de SD, y casos de AE en familiares en primer } \\
\text { grado de parentesco }\end{array}$ & $(8 / 140)$ & $5,71 \%$ \\
\hline $\begin{array}{l}\text { Parejas con un caso de SD y con antecedente de solo AE } \\
\text { Familias con más de un paciente afectado con SD en el mismo } \\
\text { núcleo familiar }\end{array}$ & $(7 / 140)$ & $5 \%$ \\
\hline $\begin{array}{l}\text { Familias con más de un paciente afectado con SD en primer } \\
\text { grado de parentesco }\end{array}$ & $(3 / 140)$ & $2,14 \%$ \\
\hline \multicolumn{1}{|c|}{ Total } & $14 / 140)$ & $2,14 \%$ \\
\hline
\end{tabular}


Resumen de las características y sus porcentajes encontrados en las 140 familias encuestadas. *SD: Síndrome de Down, †AE: Abortos espontáneos.

Solo el 5.8\% de los pacientes (7/140) se habían realizado previamente el examen de cariotipo como prueba de diagnóstico posnatal, de éstos, el $100 \%$ correspondieron a trisomías libres del cromosoma 21 , el $94 \%$ de los pacientes no se realizaron el examen de cariotipo. El 0,71\% (1/140) de los padres de familia manifestaron haberse realizado el examen de cariotipo con resultado normal (46,XX y 46,XY), mientras que el $99.29 \%$ de los padres $(139 / 140)$, no se realizaron el diagnostico citogenético.

La figura 2 muestra un ejemplo del resultado de la indagación de antecedentes familiares, en donde se expone una genealogía de una familia en cuya tercera generación se registraron dos casos de SD; uno de ellos (III:1) es un paciente de sexo femenino, con 7 años de edad y otro caso relacionado en el mismo núcleo familiar por línea materna en primer grado (III:3). De igual manera se registra un caso de aborto espontaneo en la familia. Mediante cariotipo se confirmó en el paciente III:1, una trisomía libre $(47, \mathrm{XX},+21)$, descartando una condición cromosómica heredada como responsable de la recurrencia del síndrome.

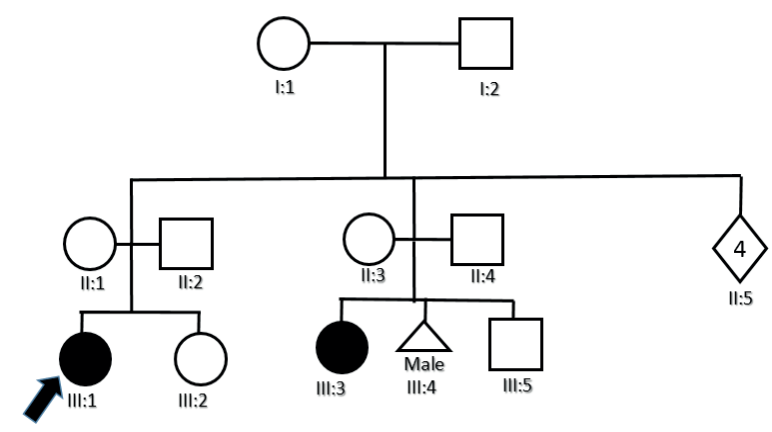

Figura 2. Árbol genealógico de una familia con caso recurrente de SD y un aborto espontaneo, en el cual se descartó una condición cromosómica responsable de la recurrencia de esta condición.

\section{DISCUSIÓN}

Al carecer Boyacá de estudios relacionados con las características poblacionales asociadas al $\mathrm{SD}$ a cualquier nivel, este estudio se convierte en un aporte en la descripción de algunas variables relacionadas con la ocurrencia del SD en el departamento, esto resulta substancial si se tiene en cuenta que el SD presenta una heterogeneidad en cuanto a sus características poblacionales, y que en que en otras partes del país éstas se han descrito claramente en este grupo de pacientes [24].

\subsection{DISTRIBUCIÓN DE CASOS DE SD EN EL DEPARTAMENTO DE BOYACÁ.}

El mayor número de casos afectados con SD se registró en Tunja, Sogamoso y Duitama, con un número de 18 a 13 casos (12,9\%-9,3\%), estas son las ciudades más pobladas del departamento (tabla 1); enseguida se registraron Moniquirá, Chiquinquirá, Garagoa, La Capilla y Aquitania, municipios con un número mediano de habitantes (54.949-21.377), presentando de 7 a 5 casos, y finalmente los municipios con menor población, que registraron el menor número de casos de pacientes afectados con SD, de 3 a $1(2,1 \%-0,7 \%)$.

\subsection{COMPORTAMIENTO POBLACION- AL DE LA EDAD MATERNA Y PATERNA}

Tras aplicar la prueba de Kolmogorov-Smirnof se pudo notar que la edad materna y paterna al momento de la gestación siguen una distribución normal de probabilidad (Niveles críticos de $0,1720,655 ;>0,05$ ) esto se debe a que el riesgo de aparición del SD aumenta con la edad materna y disminuye posteriormente al avanzar la edad y disminuir la fertilidad. Se ha documentado la manera en que la edad materna avanzada influye drásticamente en la aparición de casos de SD [25], y mundialmente se ha reconocido como el número de casos de SD aumenta a partir de los 35 años [26]. En el presente estudio el porcentaje de madres con nacimientos de SD mayores de 35 años fue de 27\% (39/139), y el porcentaje de madres con nacimientos de SD menores de 35 años fue de 71\% (100/139), y el intervalo de edad en el cual se registró el mayor número de casos fue el de 30-34 años (27,8\% del total de casos), esto puede atribuirse a que las mujeres menores de 35 años constituyen la mayoría de la población fértil [27]. En relación con esto, se ha propuesto la necesidad de implementar un monitoreo de la 
edad materna en la ocurrencia del SD, debido a que en lo que respecta a la edad materna, la tasa de variación de SD en diferentes comunidades a nivel mundial puede cambiar en un $25 \%$ según la población $[28,29,30]$.

Por su parte, se ha logrado establecer como la edad paterna avanzada constituye un factor de riesgo para tener hijos afectados por SD [31], autores atribuyen esta condición a el número de divisiones celulares mitóticas durante la espermatogénesis y su marcado aumento con la edad, comparado con la oogénesis, esto sería un argumento a favor del riesgo de mutaciones en los gametos de hombres de mayor edad, demostrándose que el aumento del daño del $\mathrm{ADN}$ en el espermatozoide de hombres entre 36 y 57 años es tres veces mayor que los hombres menores de 35 años [32], de este modo las alteraciones cromosómicas responsables del SD se dan durante la gametogénesis y al aumentar la edad de los padres aumentan también las probabilidades de que en éstos se generen anormalidades por los daños acumulados en el ADN [33].

\subsection{LA ESTRUCTURA DE LA EDAD MATERNA.}

Al comparar la edad materna registrada en el presente estudio con la registrada por diferentes estudios colombianos y latinoamericanos (Tabla 4) se pudo notar que para el quinquenio de 15 a 19 años, el presente estudio registró un menor número de casos frente a los casos registrados en los estudios de Cali [6], Pasto [7] y Guatemala [31]; esto indica que el número de mujeres entre los 15 y $\operatorname{los} 19$ años afectadas con nacimientos con SD, es mucho menor que el registrado en los estudios anteriormente nombrados, sin embargo, se pone en evidencia el número elevado de casos de $\mathrm{SD}$ en edades maternas inferiores a 30 años, pues el quinquenio de 25-29 años registra el $20,71 \%$ del total de los casos, y el quinquenio de 20-24 años, el $16,42 \%$.

Tabla 4. Porcentaje de número de casos de SD por quinquenios de edades maternas, en diferentes estudios colombianos y latinoamericanos, donde se resumen los hallazgos en cuanto a edad materna del presente estudio y otros reportes.

\begin{tabular}{|c|c|c|c|c|c|c|}
\hline & \multicolumn{6}{|c|}{ Comparación de columna de proporción } \\
\hline & $\begin{array}{l}\text { Pre- } \\
\text { sente } \\
\text { Estudio }\end{array}$ & $\begin{array}{c}\text { Ra- } \\
\text { mírez et } \\
\text { al.1996-cali } \\
{[6]}\end{array}$ & $\begin{array}{c}\text { Hernán- } \\
\text { dez et al. } \\
2006 \text { - Pasto } \\
\text { [7] }\end{array}$ & $\begin{array}{r}\text { Acevedo } \\
2002 \text { Guate- } \\
\text { mala [31] }\end{array}$ & $\begin{array}{l}\text { Sánchez et } \\
\text { al. 2000-Ca- } \\
\text { raccas } \\
\text {-Venezuela } \\
\text { [29] }\end{array}$ & $\begin{array}{c}\text { Astete et } \\
\text { al.1991- San- } \\
\text { tiago de Chile } \\
{[40]}\end{array}$ \\
\hline $\begin{array}{c}\text { Tamaño de la } \\
\text { muestra }\end{array}$ & 140 & 298 & 49 & 533 & 15 & 257 \\
\hline $\begin{array}{c}\text { Edad } \\
\text { materna en } \\
\text { quinquenios }\end{array}$ & $(\%)$ & $(\%)$ & $(\%)$ & $(\%)$ & $(\%)$ & $(\%)$ \\
\hline de 15-19 & 2,14 & 9,52 & 16,3 & 12,38 & 0 & 8,39 \\
\hline de $20-24$ & 16,42 & 20,63 & 8,2 & 17,45 & 6,67 & 18,18 \\
\hline de $25-29$ & 20,71 & 19,04 & 6,1 & 16,32 & 33,3 & 14,69 \\
\hline de $30-34$ & 27,85 & 14,28 & 26,5 & 19,32 & 26,27 & 17,48 \\
\hline de $35-39$ & 19,28 & 19,04 & 26,5 & 20,83 & 33,3 & 24,48 \\
\hline de $40-44$ & 7,59 & 12,69 & 12,6 & 11,26 & 0 & 17,78 \\
\hline de $45-49$ & 5,14 & 4,76 & 2 & 1,88 & 0 & 0 \\
\hline
\end{tabular}

Se encontró, además, que para el quinquenio de 30 a 34 años, en la presente investigación se registró un mayor número de casos de mujeres con nacimientos de SD que en el estudio de Cali [6] (tabla 4), sin embargo, hay que tener en cuenta que estas comparaciones pueden no reflejar com- 
portamientos reales debido a la imposibilidad de comparar estadísticamente los resultados del presente estudio con los reportados por diferentes autores por el uso de metodologías y tamaños muestrales disímiles.

A futuro se recomienda implementar estudios a nivel departamental y nacional que permitan ampliar e identificar factores de riesgo para el SD, por ejemplo, se ha documentado cómo la etnicidad puede influir en la aparición de la patología al determinar que las madres de origen hispánico tienen mayor riesgo de tener hijos con SD [32, 33]; habría que determinar si el grado de influencia de la composición étnica de esta región del país tiene influencia hispánica, indoamericana e incluso africana [34, 35].

\subsection{SD RECURRENTE EN EL NÚCLEO FAMILIAR}

Según resultados del análisis de genealogías y del análisis discriminante, en el 4,28\% (6/140) de las familias encuestadas se presentaron varios casos de SD en el mismo núcleo familiar, el cariotipo realizado a las personas descartó la presencia de alteraciones heredables relacionadas con el SD familiar, por lo que en la asesoría genética se atribuyeron estos casos a la edad materna avanzada [35].

\subsection{ABORTOS ESPONTÁNEOS}

En lo que respecta a los abortos espontáneos, se evidenció que el 10,15\% de las familias (15/140) presentaron esta condición (tabla 2); esto concuerda con lo mostrado por el estudio de Corona A, et al. [12], que evaluó un total de 48 familias en cuyos integrantes había casos de SD, y encontró abortos en un $9.11 \%$ de las familias participantes; es de notar que no se presentaron marcadas diferencias entre los dos estudios. Existe un mayor riesgo de abortos en familias con casos de SD que en familias que no presentan esta condición; esto ratifica la necesidad de que estas familias tengan un seguimiento en sus gestaciones y que participen de una asesoría genética que les dé a conocer los riesgos que existen, por la condición de la patología [36, 37].

El 66\% de las causas de abortos fueron idiopáticas (tabla 2), seguidas por causas anatómicas $(18,1 \%)$, endocrinológicas $(11,1 \%)$ e inmunológicas (4,7\%), a ningún producto de aborto se le realizó el examen de cariotipo, lo que impidió demostrar la existencia de un factor genético en dichas pérdidas, el cual, teóricamente, es responsable del $50 \%$ de los abortos espontáneos [38]. En las madres que sufrieron eventos de abortos espontáneos se encontró que el rango de edades estuvo entre los 26 y los 39 años, con un mediana de edad de 33 años; esto concuerda con lo reportado por diferentes autores [39], pues se ha establecido un riesgo mayor de padecer eventos de abortos espontáneos en gestaciones con edades superiores a 30 años, sin olvidar que algunas de estas madres tenían el antecedente de hijos con $\mathrm{SD}$ [40].

\subsection{EXAMEN DE CARIOTIPO EN LAS PERSONAS AFECTADAS CON SD}

Los pacientes afectados con SD a quienes se les había realizado el examen de cariotipo fueron 7

(5.8\% de los casos encuestados); al igual, se presentó un bajo porcentaje de familias que cuentan con un estudio citogenético en alguno de sus miembros, es así como solo una pareja de padres, es decir, el $0.72 \%$ del total de las familias encuestadas (1/140), se realizó el diagnóstico, esto reafirma que en el departamento de Boyacá, el examen de cariotipo no era rutinariamente utilizado como apoyo diagnóstico para el estudio de anomalías cromosómicas como el SD, debido al desconocimiento de esta prueba por la población, y a que no era disponible en el sistema de salud colombiano [41]. Con los Acuerdos 028 y 029 de 2011, la Comisión de Regulación en Salud reglamentó la inclusión en el Plan Obligatorio de Salud (POS) el cariotipo como examen de apoyo que deben brindar las Entidades Promotoras de Salud del país [42]; se espera que con el apoyo de las Secretarías de Salud y Educación del departamento, los estudios citogenéticos se empiecen a implementar y difundir, pues a la fecha su disponibilidad sigue siendo baja. 


\section{CONCLUSIONES}

El estudio permitió realizar un análisis descriptivo de algunas de las variables asociadas con la ocurrencia del SD en 43 localidades del departamento de Boyacá, constituyéndose en un primer acercamiento al estudio del comportamiento poblacional del SD en Boyacá. Se ratifica la influencia de la edad materna en la aparición de casos con SD, pues el porcentaje de madres con nacimientos de SD mayores de 35 años fue de 27\% (39/139), aunque el mayor número de casos con SD nacieron de madres en edades de $30-35$ años $(27,8 \%$ del total de casos), pero esto se puede atribuir a que las mujeres menores de 35 años constituyen la mayoría de la población fértil.

En Boyacá resulta necesario adelantar estudios a nivel poblacional, en los que se establezca la incidencia y se estudien las demás variables relacionadas con el SD, para caracterizar la población con SD del departamento. Es necesario fomentar estudios citogenéticos que apoyen la accesibilidad de este procedimiento; además, se recomienda establecer la asesoría genética como parte integral del diagnóstico del SD en el departamento, la cual permitirá a las familias conocer el origen y el riesgo de recurrencia del SD en sus familias y así apoyarlas en la toma de decisiones informadas.

\section{AGRADECIMIENTOS}

Al doctor JORGE HUMBERTO CASTILLO SILVA. A la secretaria de educación del departamento de Boyacá, a las fundaciones sin ánimo de lucro FUNDOWN, SUPERAR, ASDEPAL, UAI PROGRAMA RBC-Duitama, AULA TECNOLOGICA CULTURAMA, UAI PROGRAMA RBC-Paipa, FUNDINES, FUNDIFERENTE. A las instituciones educativas SUSANA GUILLEMIN, CAMPO ELIAS CORTES, JOSE MARIA SILVA SALAZAR, MARIA AUXILIADORA, TÉCNICO AGROPECUARIA, COLEGIO INTEGRADO, JUANA CAPORAL, SANTOS GUTIERREZ, EL ESPINO y demás instituciones y personas que de una u otra forma participaron de la investigación. A la dirección de investigaciones de la UPTC (DIN) por el respaldo para realizar la investigación (código del proyecto SGI 854).

\section{CONFLICTO DE INTERESES Y FINANCIACIÓN}

Los autores del presente artículo declaran que no hay ningún conflicto de intereses en el desarrollo del mismo. La financiación de la investigación se obtuvo de recursos del grupo de investigación GEBIMOL de la Universidad Pedagógica y Tecnológica de Colombia.

\section{BIBLIOGRAFÍA}

[1] L. Cifuentes, and J. Nazer, "Epidemiología de malformaciones congénitas en Chile", Acta biol. Colomb., vol. 13, no. 3, p. 7, 2008.

[2] R. L. Nussbaum, R. R. Mclnnes, and H. F. Willard, Thompson y Thompson Genética en Medicina, $7^{\text {a }}$ edición Elsevier-Masson, 2008, ISBN 978-84-458-1870-1, pp. 75, 81.

[3] A. D. Carothers, C. A. Hectch, and E. B. Hook, "International variation in reported livebirth prevalence rates of Down Sindrome, adjusted for maternal age", J. Med. Genet., 36, pp. 386-393, 1999.

[4] J. Nazer, and L. Cifuentes, "Malformaciones congénitas en Chile y Latino América: Una visión epidemiológica del ECLAMC del período 1995-2008", Rev. Med. Chile, no. 139, pp. 72-78, 2011.

[5] C. Valencia, A. Naranjo, G. Giraldo, and E. Castaño, "Prevalencia al nacimiento deSíndrome de Down en la ciudad de Manizales (Caldas-Colombia) durante el período 20042005”, Biosalud, vol. 7, pp. 69-74, 2008.

[6] R. Ramírez, C. Isaza, and M. Gutiérrez, "La incidencia del síndrome de Down en Cali, Colombia", Med., vol. 27, no. 3-4, pp. 13842, 1996.

[7] H.I. Hernández, and R. Manrique, "Prevalencia de síndrome de Down en CEHANI-ESE, San Juan de Pasto, Colombia. 1998-2003", NovaPublicación Científica, vol. 4 , no. 5, pp. 50-56, 2006. 
[8] N.E. Lamb, K. Yu, J. Shaffer, E. Feingold, S.L. Sherman, "Association between maternal age and meiotic recombination for trisomy 21", Am. J. Hum. Genet., vol. 76, no. 1, pp. 91-99, 2005.

[9] J. C. Montoya, J. M. Satizábal, F. García, A. Sánchez, "Prospectiva y comprensión bioquí- mica del síndrome de Down", El hombre y la máquina, no. 30, pp. 118-129, 2008.

[10] A.S. Brown, E. Feingold, K. W. Broman, S.L. Sherman, "Genomewide variation in recombination in female meiosis: a riskfactor for nondisjunction of chromosome 21", Hum. Molec. Genet. vol. 9, pp. 515-523, 2000.

[11] B. Hämmerle, J. Colonques, E. Vera, J. Chulia, F. J. Tejedor, "Bases moleculares de las neuropatologías del síndrome de Down: implicación del gen Minibrain", Mapfre Medicina, vol. 14, pp. 210-216, 2003.

[12] A. Corona, T. García, S. Totsuka, J. Corona, L. Bobadilla, and E. Corona, "Frecuencia de abortos en familias con un caso de síndrome de Down", Bol. méd. Hosp. Infant. Méx., vol. 51, no. 7, pp. 463-467, 1994.

[13] M. Sánchez, and Z. Corredor, Determinación de anormalidades cromosómicas en pacientes con esquizofrenia de Boyacá, Colombia. Tesis de pregrado. Universidad Pedagógica y Tecnológica de Colombia, Tunja, 2009.

[14] J. Corretger, A. Serés, J. Casaldàliga, and K. Trias, Síndrome de Down: aspectos médicos actuales, Ed. Masson, para la Fundación Catalana del Síndrome de Down, 2005.

[15] S.B. Freeman, E.G. Allen, C.L. Oxford-Wright, S.W. Tinker et al., "The National Down Syndrome Project: design and implementation", Public. Health. Rep., no. 122, pp. 62-72, 2007.

[16] R. Resta, "Changing demographics of advanced maternal age (AMA) and the impact on the predicted incidence of Down syndrome in the United States: Implications for prenatal screening and genetic counseling", American Journal of Medical Genetics Part A, vol. 133A, pp. 31-36, 2005.

[17] B. Trimble, and P. Baird, "Maternal Age and Down Syndrome: Age-specific incidence rates by single-year intervals", American Journal of Medical Genetics, vol. 2, no. 1, pp. 1-5, 2005.

[18] H. Fisch, K. Hyun, R. Golde, T. Hensle, C. Olsson, G. Liberson, "The Influence of Paternal Age on Down Syndrome", The Journal of Urology, vol 169, pp. 2275-2278, 2003.

[19] J.K. Morris, E. Alberman.Trends in Down's Syndrome Live Births and Antenatal Diagnoses in England and Wales from 1989 to 2008: Analysis of data from the National Down Syndrome Cytogenetic Register BMJ, vol. 339, pp. b3794, 2009.

[20] B. Kallen, P. Mastroiacovo, and E. Robert, "Major congenital malformations in Down Syndrome", Am. J Med. Genet., vol. 65, pp. 160-166, 1996.

[21] E. Hook, and P.K. Cross, "Spontaneous abortion and subsequent Down Syndrome Livebirth", Human Genetics, vol. 64, no. 3, pp. 267- 270, 1983.

[22] T. Ikeuchi, "Inhibitory effect of ethidium bromide on mitotic chromosome condensation and its application to high resolution chromosome banding". Citogenet Cell Genet, 1984; 38: 56-61. En: Barch MJ, Knutsen T, Spurbeck JL. The AGT Cytogenetics Laboratory Manual. Philadelphia: Editorial Lippincott-Raven, pp. 7-171, 1997.

[23] L.G. Shaffer, N. Tommerup. ISCN: An International System for Human Cytogenetic Nomenclature (Cytogenetic \& Genome Research). 2013.

[24] C.P. Torfs, and R.E. Christianson, "Anomalies in Down Syndrome Individuals in a 
Large Population-Based Registry”, Am. J. Med. Genet., vol. 77, pp. 431-438, 1998.

[25] H. García, G. Salguero, J. Moreno, C. Arteaga, and A. Giraldo, "Frecuencia de anomalías congénitas en el Instituto Materno Infantil de Bogotá", Biomédica, 23: 161-172, 2003.

[26] N. J. Roizen, and D. Patterson, “Down's Syndrome”, Lancet, 361: 1281-9, 2003.

[27] J. García-Rodríguez, and N. Ramírez, "Factors Affecting How Long Exclusive Breastfeeding Lasts", Rev. Salud Pública, vol. 10, no. 1, pp. 71-84, 2008.

[28] M. A. Canfield, M.A. Honein, N. Yuskiv, J. Xing, C.T. Mai, J.S. Collins et al., "National stimates and race/ethnic-specific variation of selected birth defects in the United States, 1999- 2001. Birthdefects Res A Clin Mol Teratol, vol 76, pp. 757-756, 2006.

[29] A. J. Sánchez, W. Lorizio, I. M. Durán, and R. L. Romero, Down's Syndrome. Epidemiology Characterization, República Bolivariana de Venezuela, La Universidad del Zulia, Facultad de Medicina, Hospital General, Dr. Adolfo D’empaire, 2000.

[30] M. Kim, H. Nan, S. Jeong, Y. Jung, and S. Kyung, "Prevalence of Birth Defects in Korean Livebirths, 2005-2006", J. Korean Med. Sci., 27: 1233-1240, 2012.

[31] M. A. Acevedo, and C. I. Howard, "Aspectos clínicos del síndrome de Down. Revista Pediatría Universitaria, 1(1): 48, jul-ago-sep, 2002.

[32] A. Aleyasin, M. Ghazanfari, and G. S. Mohammad, "Application of Molecular DNA Markers (STRs) in Molecular Diagnosis of Down Syndrome in Iran", Journal of Sciences, Islamic Republic of Iran, 15(2): 103-108, 2004.

[33] S.L. Sherman, S. Freeman, E.G. Allen, and N. Lamb, "Risk factors for nondisjunction of trisomy 21", Cytogenet. Genome Res., vol. 111, pp. 273-80, 2005.

[34] F. Lizcano, "Composición étnica de las tres áreas culturales del continente americano al comienzo del siglo XXI". Convergencia. Centro de Investigación en Ciencias Sociales y Humanidades, UAEM, vol. 38, pp. 185-232, 2005

[35] M. Dundar, A. Caglayan, K. Arslan, and Y. Ozkul, "Down syndrome like appearance with a novel de novo translocation $\mathrm{t}(6 ; 21)$ (q21;q13)", Indian J. Med. Res., vol. 128, pp. 666-668, 2008.

[36] F. Álvarez-Nava, M. Soto, A. Morales, A. Rojas, K. Urdaneta, and J. Cañizález, "Prospective prenatal serum screening for Down síndrome in Venezuela", International Journal of Gynecology and Obstetrics, vol. 103, pp. 241-245, 2008.

[37] C. P. Griebel, "Management of abortion spontaneous". Am Fam Phys., vol. 72, pp. 1243-1250, 2005.

[38] M. Quiroga de Michelena, A. Díaz, D. Paredes, O. Rodríguez, E. Quispe, and E. Klein, "Estudio cromosómico en el aborto espontáneo y su aplicación clínica", Rev. Per. Ginecol. Obstet., vol. 53, no. 2, pp. 124129, 2007.

[39] E.E. Castilla, and I. M. Orioli, "The Latin American CollaborativeStudy of Congenital Malformation", Community Genetics, 7: 76-94, 2004.

[40] C. Astete, R. Youlton, S. Castillo, C. Be, and V. Daher, "Análisis clínico y citogenético en 257 casos de síndrome de Down”, Rev. Chil. Pedlatr., vol. 62, no. 2, pp. 99-102, 1991.

[41] J. Masis D., and M. Castellanos, "Caracterización de los recobros por tutelas y medicamentos no incluidos en los planes obligatorios de salud", Revista Gerencia y Políticas de Salud, 2004. 
E. J. Vergara Estupiñán et. al.

[42] Colombia, Ministerio de Salud y Protección Social, Acuerdo 029 de 28 de diciembre de 2011, por el cual se actualiza y aclara integralmente el Plan Obligatorio de Salud, 2012. 